\title{
Commissural Malalignment as a predictor of coronary artery abnormalities in patients with transposition of great arteries
}

Mohamed Al Nasef ${ }^{1,2}$ (D) Mohammed H. Alghamdi ${ }^{1,3}$, Maria L. Bello Valls ${ }^{1}$, Ahmed M. Zahranii ${ }^{4}$, Ali AlAkfash ${ }^{5}$, Husam I. Ardah ${ }^{6,7}$, Obayda M. Diranneya ${ }^{1}$ and Fahad Alhabshan ${ }^{1,6,7^{*}}$

\begin{abstract}
Background: In patients with transposition of the great arteries (TGA), commissural malalignment (CM) between semilunar valves may be associated with abnormal coronary (CA) pattern. We intend to assess the degree of CM with incidence of unusual CA anatomy.

Methods: We proposed a ratio to measure the distance of both ends of the anterior facing sinuses of the pulmonary valve from the facing commissure of the aortic valve. We labeled it as D1 and D2 distance. A ratio (C ratio) of the smaller distance (either D1 or D2 whichever is shorter) over the sum of both D1 and D2 was taken (D1 or D2 whichever is shorter / D1 + D2). We related this ratio with the incidence of the unusual CA anatomy in D-TGA patients.

Results: We had a total of 158 patients. We defined the point beyond which the C-Ratio becomes significantly associated with abnormal coronary artery pattern, this represents the median effective level (EL50). The EL50 of the C-Ratio was found to be equal to 31\% (0.31). The prediction revealed that the CA pattern would most probably be usual when there is a minor commissural malalignment (C-Ratio less than the EL50) and most probably be unusual when there is a major malalignment (C-Ratio is greater than the EL50). The sensitivity was $71 \%$ and the specificity $88 \%$ ( $p$-value $<0.0001$ ).
\end{abstract}

Conclusions: The C-Ratio helps to categorize the degree of CM as minor (less than 0.31) or major (more than 0.31). A higher C-Ratio predicts a higher incidence of unusual CA pattern.

Keywords: Transposition of great arteries, Commissural malalignment, Coronary abnormality, Arterial switch operation

\footnotetext{
* Correspondence: habshanf@ngha.med.sa

${ }^{1}$ Cardiac Sciences Department, Ministry of the National Guard - Health Affairs, Riyadh, Saudi Arabia

${ }^{6}$ King Abdullah International Medical Research Center, Riyadh, Saudi Arabia

Full list of author information is available at the end of the article
}

(C) The Author(s). 2020 Open Access This article is licensed under a Creative Commons Attribution 4.0 International License, which permits use, sharing, adaptation, distribution and reproduction in any medium or format, as long as you give appropriate credit to the original author(s) and the source, provide a link to the Creative Commons licence, and indicate if changes were made. The images or other third party material in this article are included in the article's Creative Commons licence, unless indicated otherwise in a credit line to the material. If material is not included in the article's Creative Commons licence and your intended use is not permitted by statutory regulation or exceeds the permitted use, you will need to obtain permission directly from the copyright holder. To view a copy of this licence, visit http://creativecommons.org/licenses/by/4.0/ The Creative Commons Public Domain Dedication waiver (http://creativecommons.org/publicdomain/zero/1.0/) applies to the data made available in this article, unless otherwise stated in a credit line to the data. 


\section{Introduction}

Transposition of the great arteries (TGA) is the one of the most common cyanotic congenital heart lesions that presents in the neonatal period [1]. Prior reports on factors affecting the outcome of the ASO demonstrated that abnormal CA patterns, such as single right, inverted coronaries and intramural course of the $\mathrm{CA}$, are associated with increased risk of early mortality [2-10]. With the current improved surgical skills, the outcome in patients with uncommon CA pattern is similar to those with usual CA pattern $[9,11,12]$.

The presence of malalignment between the commissures of the semilunar valves has been shown to be associated with abnormal CA pattern which may affect the surgical approach during ASO [13]. However, it is not known what degree of commissural malalignment (CM), in patients with TGA, would result in an abnormal CA pattern. Furthermore, no clear definition was set for the so called "major CM" and "minor CM", since it was always a subjective terminology. In addition to that, it was not clear whether CM in these patients would have any perioperative or postoperative consequences. Therefore, the primary aim of our study is to provide a clear quantitative definition to describe the minor and major CM, and to investigate its relationship with the incidence of unusual CA pattern in patients with TGA. The secondary aim is to explore the effect of minor and major CM on the surgical outcome of these patients.

\section{Methods}

Our study is a retrospective cohort study. All patients with simple TGA who presented to King Abdulaziz Cardiac Centre (KACC), Riyadh, Saudi Arabia during the period between June 2005 till December 2017 were included. Patients who had complex TGA, defined as association with subvalvular or supravalvular aortic stenosis, coarctation of aorta, Taussig Bing anomaly, pulmonary stenosis, unusual great artery relationship were excluded. Patients with suboptimal echocardiography (Echo) images were also excluded from our study.

The demographic and surgical outcome data were collected from our electronic database, BESTCare ((Bundang hospital Electronic System for Total Care), Seoul, South Korea)). Echo data was collected from our digitally stored images and reports in Xcelera system (Philips Medical Systems, Best, Netherlands). All the patients in our list have undergone Echo with standardized protocols as per the American society of echocardiography (ASE) guidelines [14]. The pattern of CA anatomy in patients with TGA was carefully identified in the parasternal short axis views as described by the ASE report [14]. Optimal images were defined as both semilunar valves seen in cross sectional views and both coronary arteries origins are visualized. The CA pattern was classified as
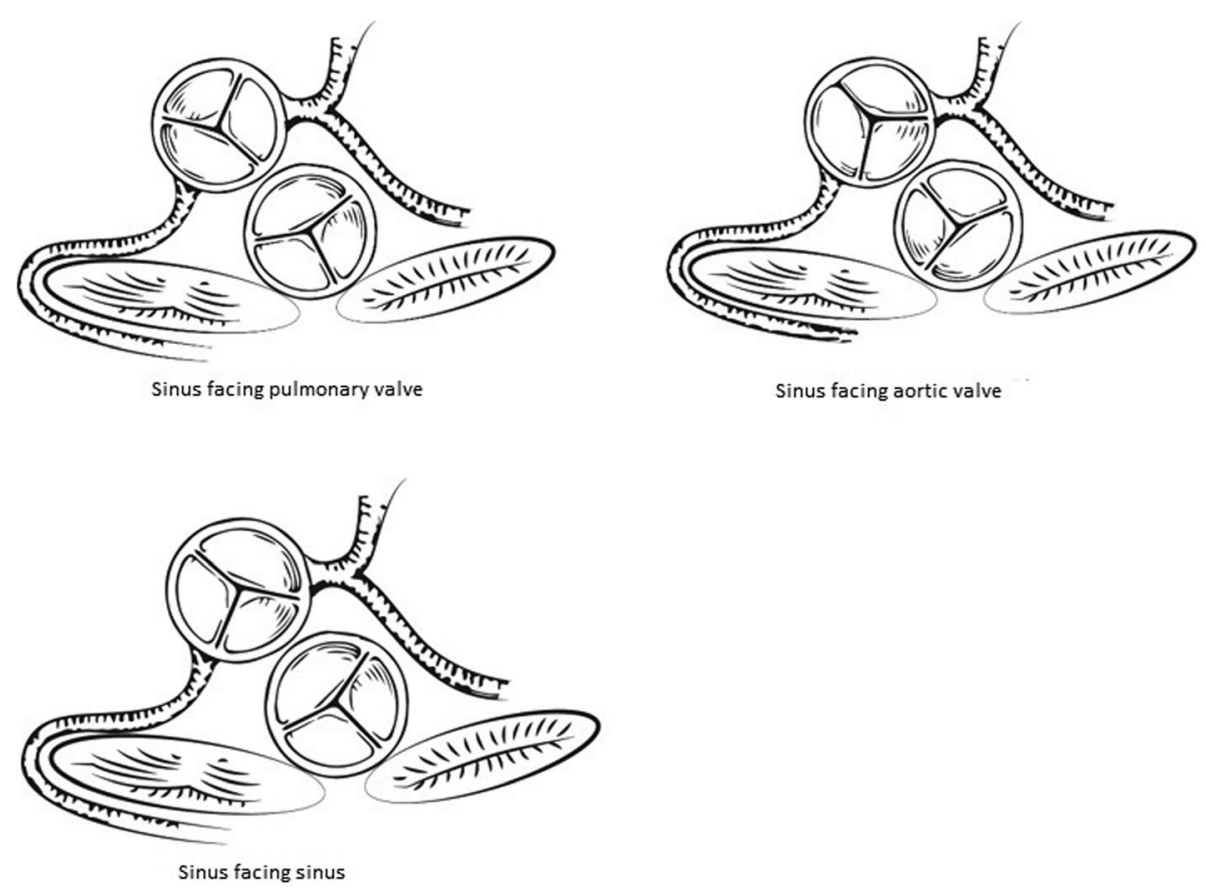

Fig. 1 Types of commissural malaligment. Sinus facing pulmonary valve:-When commisure of the aortic valve faces the sinus of the pulmonary vallve. Sinus facing aortic valve:-When the commisure of the pulmonary valve faces the sinus of the aortic valve. Sinus facing sinus:-When both sinuses faces each other 
per the scoring system published by Wernovsky et al. and others $[8,15]$. CA pattern was also confirmed by the surgeons intraoperative assessment. We classified the pattern of CM by referring it to possible positions of the sinuses of the valves in relation to each other such as sinus facing aortic valve, sinus facing pulmonary valve or sinus facing sinus as shown in Fig. 1. This comes partly in line with the category of CM that was published by Kim et al. in 2003 [13]. Sinus facing aortic valve is described as the commissure of the pulmonary valve lying opposite to any part of the sinus of the aortic valve. Sinus facing pulmonary valve is described as commissure of the aortic valve facing any part of the pulmonary valve. Sinus facing sinus is described in cases where both sinuses face each other.

We propose in this study a new ratio that is expected to differentiate between minor and major $\mathrm{CM}$. This ratio is obtained by measuring the distance of both ends of the anterior facing sinuses of the pulmonary valve from the facing commissure of the aortic valve, as shown in Fig. 2. The distance from the commissure of the aortic valve to the right sided commissure of the pulmonary valve is labeled as D1, while the distance from the aortic commissure to the left sided commissure of the pulmonary valve is labeled as D2. In patients with side by side relationship of semilunar valves, D1 represents the measurement from the commissure of the aortic valve to the inferior commissure of the pulmonary valve, while D2 represents the measurement from the commissure of the aortic valve to the superior commissure of the pulmonary valve. We propose a commissural ratio (C-Ratio) where D1 or D2 (whichever is shorter) is divided by the sum of D1 and D2. We have used the C-Ratio to assess the degree of $\mathrm{CM}$ and to differentiate between the major and minor one. In addition to that, we have used this ratio to study the relation between $\mathrm{CM}$ and the incidence of unusual CA pattern in patients with TGA. We have defined the point beyond which the C-Ratio becomes significantly associated with abnormal CA pattern, this represents the median effective level (EL50).

The data is expressed as means and standard deviations for normally distributed data and as medians with ranges for skewed data. $P$ value of less than 0.05 is considered as significant. The statistical analyses were performed using SPSS for Windows, Version 16.0. Chicago, SPSS Inc.

\section{Results}

During the study period, 197 patients were diagnosed with TGA. One hundred fifty eight patients fit the inclusion criteria while the rest were excluded as per our exclusion criteria. The mean weight of the patients was $3.4 \mathrm{~kg}( \pm 1.4)$ (Table 1). One hundred three patients (65\%) had TGA with intact ventricular septum (IVS),
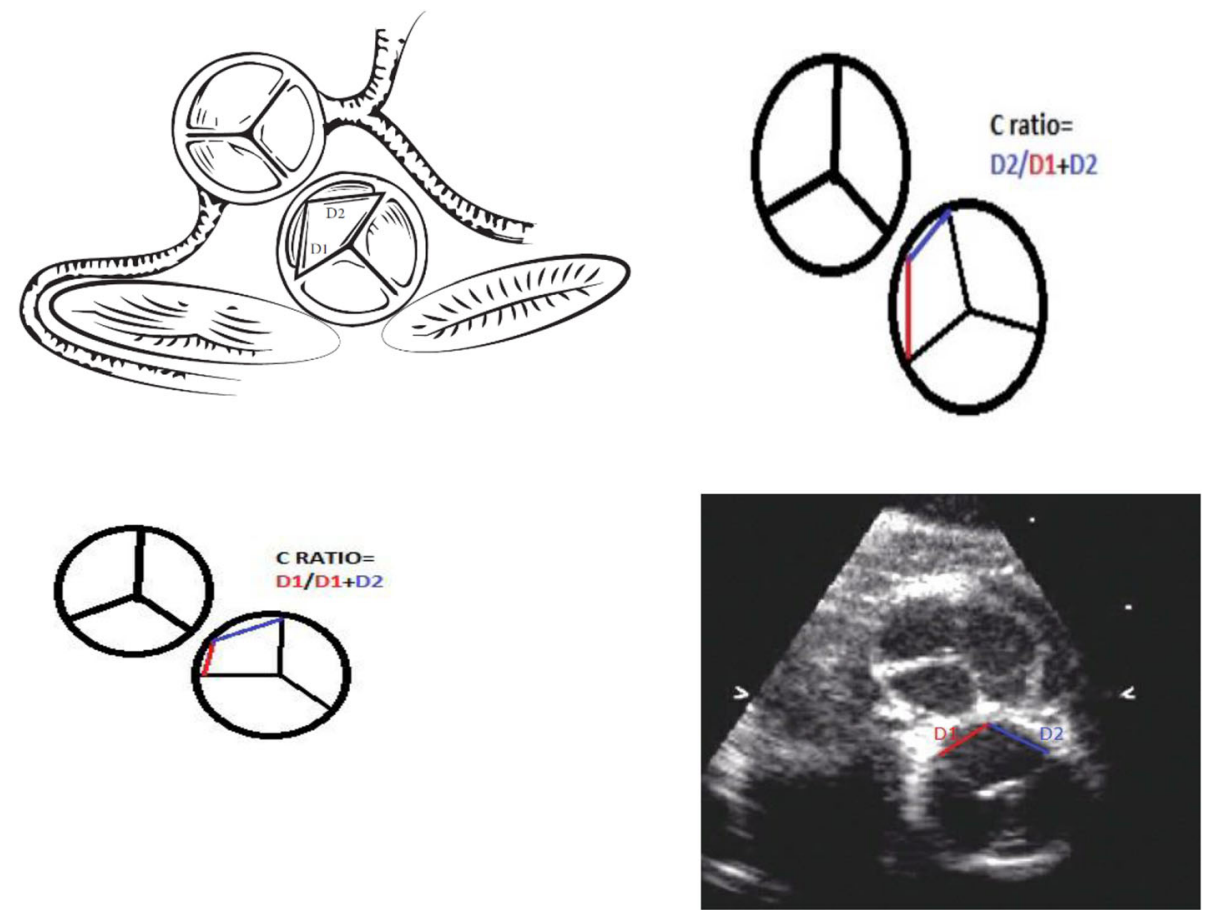

Fig. 2 Measuring the C-Ratio. The Distance from the anterior commissure to the right sided commissure of the pulmonary valve is labelled ad D1, while the distance from the anterior commissure to the left sided commissure of the pulmonary valve is labelled as D2. C-Ratio = D1 or D2 whichever is shorter divided by D1 + D2 
while the rest (35\%) had TGA with VSD. One hundred thirteen patients $(72 \%)$ had usual CA pattern while 45 (28\%) had unusual CA anatomy. Left circumflex CA arising from the right coronary artery was the most common unusual CA pattern in our patient population (40\%) (Table 2). 50/103 patients with TGA and IVS (49\%) and 27/55 patients with TGA + VSD (60\%) had commissural malalignment. All except 4 of our patients had a sinus facing pulmonary valve. Six patients had an intramural course, and most of these patients (4 out of 6) had a single right CA pattern with left intramural coronary artery. Interestingly, all patients with intramural course of CAs had commissural malalignment.

We defined the point beyond which the C-Ratio becomes significantly associated with abnormal CA pattern (EL50) which was found to be equal to $31 \%(0.31)$. Out of 158 patients, $48(30 \%)$ had a C-Ratio more than 0.31 and were considered to have major commissural malalignment, while 110 patients (70\%) had a C-Ratio less than 0.31 and were considered to have either minor malalignment or no malalignment. We have crossclassified the binary response (usual versus unusual CA pattern) with a prediction of whether the patient has a major or minor/no commissural malalignment. Out of the 48 patients who had major malalignment, 32 (67\%) had unusual CA pattern, while 16 (33\%) had usual CA pattern. On the other hand, 97 patients $(88 \%)$ of the group with minor malalignment or no malalignment had usual CA pattern, while only 13 (12\%) had unusual CA pattern. The prediction revealed that the $\mathrm{CA}$ pattern would most probably be usual when there is a minor $\mathrm{CM}$ or no CM (C-Ratio less than the EL50) and most probably be unusual when there is a major malalignment (C-Ratio is greater than the EL50). The sensitivity was $71 \%$ and the specificity was $88 \%$. A logistic regression analysis was done to model the probability of an unusual CA pattern with the C-Ratio as a linear predictor. It revealed that the C-Ratio is statistically significant at the 0.05 level ( $p$-value $<0.0001)$. The estimated odds ratio is 1.13 for every $1 \%$ increase in the C-Ratio with a 95\% confidence interval of (1.087-1.175). Thus, there is a $13 \%$ increase in the odds for every 0.01 increase in the

Table 1 Demographics

\begin{tabular}{llll}
\hline Characteristics & $\begin{array}{l}\text { Usual CA } \\
(\boldsymbol{n}=\mathbf{1 1 3})\end{array}$ & $\begin{array}{l}\text { Unusual CA } \\
(\boldsymbol{n}=\mathbf{4 5})\end{array}$ & $\boldsymbol{P}$ value \\
\hline Weight $(\mathrm{kg})( \pm \mathrm{SD})$ & $3.3 \pm 1.4)$ & $3.4( \pm 0.8)$ & 0.5 \\
Height $(\mathrm{cm})( \pm \mathrm{SD})$ & $52 \pm \underline{ \pm})$ & $52 \pm 20)$ & 0.5 \\
Gender & & & 0.8 \\
$\quad$ Males & $77(68 \%)$ & $30(66 \%)$ & \\
$\quad$ Females & $36(32 \%)$ & $15(34 \%)$ & \\
TGA IVS & $75(66 \%)$ & $28(62 \%)$ & 0.6 \\
TGA VSD & $38(34 \%)$ & $17(38 \%)$ & \\
\hline
\end{tabular}

Table 2 Distribution of the coronary artery patterns

\begin{tabular}{|c|c|c|}
\hline \multicolumn{2}{|c|}{ Coronary artery anatomy } & \multirow{2}{*}{$\begin{array}{l}\text { Frequency (\%) } \\
113(72 \%)\end{array}$} \\
\hline Usual & & \\
\hline \multirow[t]{6}{*}{ Unusual } & & $45(28 \%)$ \\
\hline & $1 \mathrm{LAD} 2 \mathrm{RC} \times(1$ intramural) & $18(40 \%)$ \\
\hline & 1LCxR (1 intramural) & $11(24 \%)$ \\
\hline & 2RLCx (4 intramural) & $10(23 \%)$ \\
\hline & 1LRCA 2CX & $3(6.5 \%)$ \\
\hline & 1R 2LCX & $3(6.5 \%)$ \\
\hline
\end{tabular}

Abbreviations

1: Sinus one, 2: Sinus two, LAD Left anterior descending, $C x$ Circumflex coronary artery, RCA Right coronary artery

C-Ratio. Figure 3 demonstrates the receiver operating characteristic (ROC) curve and shows that the area under the curve is 0.90 which is significant.

We analyzed the burden of the abnormal CA pattern on the surgical approach and found that the surgical bypass time was significantly shorter in patients with usual CA pattern compared to those with unusual CA pattern, $P<0.05$ (Table 3). We also analyzed the medium-term postoperative outcome in relation to the $\mathrm{CM}$ and found that the left ventricle end-diastolic dimension (LVEDd) was significantly smaller in patients with minor/no malalignment at 6 months follow-up compared to patients with major malalignment, $P<0.05$. However, there was no statistically significant difference in the ejection fraction or shortening fraction in the postoperative assessment within the first 6 months. Furthermore, there was no statistically significant difference in the hospital stay period between the two groups.

\section{Discussion}

The ASO is the standard surgical treatment for patients with TGA. Unfavorable pattern of CA anatomy was reported to be an important risk factor affecting its outcome. Various surgical techniques were described for the transfer of the CA in cases of malaligned commissures to avoid jeopardizing the coronary blood flow [5-7]. In our study, patients with TGA and VSD appeared to be more prone to CM compared to patients with TGA and IVS, nevertheless this did not reach a statistically significant $P$ value, but there was a clear trend towards this observation. In line with the previously published reports [15], we have found that the left circumflex CA arising from the RCA is the most common anomaly observed. All of our patients with an intramural course of CAs had CM, albeit this was not statistically significant due to the small number (6 patients).

The relationship between the unusual CA pattern and CM in patients with TGA was described by Kim et al. in $2003[13,16]$. They indicated that CM was a potential 


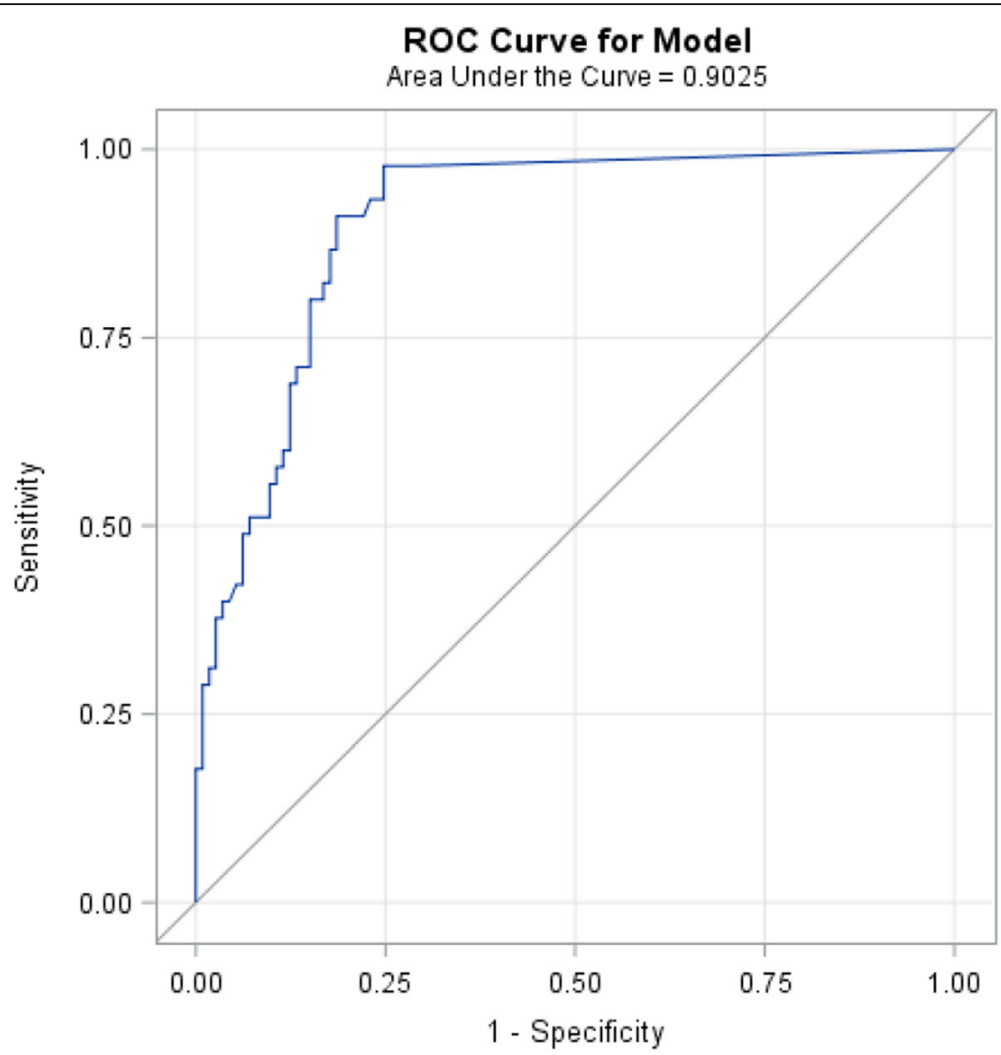

Fig. 3 Reciever operating Characterisitc (ROC) curve for the C-Ratio

risk factor for having an unusual CA pattern, but there was no clear definition for the major and minor malalignment, and their report was based on a small sample volume (13 patients).

To the best of our knowledge, our study has the largest number of patients to assess the $\mathrm{CM}$ and its relation to the CA pattern. After repeated attempts to measure the degree of rotation between the two semilunar valves, we were able to propose the C-Ratio as a new parameter which is very effective in differentiating between the major and minor malalignment as described in details in the methods section and shown in Fig. 2. We have used the C-Ratio to assess the degree of CM and to differentiate between the major and minor malalignment using the median effective level (EL50). This was defined at 0.31 above which patients were labeled as having major malalignment, while patients who had a C-Ratio less than 0.31 were labeled as having minor malalignment or having no malalignment.

In our study, two thirds (67\%) of the patients with major CM (C-Ratio above 0.31) had unusual CA pattern, while $88 \%$ of the patients with minor malalignment had usual CA pattern (C-Ratio less than 0.31). The sensitivity was $71 \%$, specificity $88 \%$ and the $P$ value was $<0.0001$.

Table 3 Surgical Outcome

\begin{tabular}{|c|c|c|c|}
\hline & $\begin{array}{l}\text { USUAL } \\
(n=113)\end{array}$ & $\begin{array}{l}\text { UNUSUAL } \\
(n=45)\end{array}$ & $P$ value \\
\hline Age at surgery (days $( \pm$ SD)) & $30( \pm 102)$ & $27( \pm 33)$ & 0.1724 \\
\hline Hospital stay (days $( \pm$ SD)) & $31(+56)$ & $33( \pm 47)$ & 0.5543 \\
\hline Bypass time & $150( \pm 58)$ & $172( \pm 63)$ & 0.0411 \\
\hline Cross clamp time & $88( \pm 36)$ & $96( \pm 35)$ & 0.2924 \\
\hline FS (Fractional shortening) 6 months after surgery & $36 \%( \pm 9)$ & $36( \pm 15)$ & 0.3062 \\
\hline EF (Ejection fraction) 6 months after surgery & $66( \pm 10)$ & $63( \pm 13)$ & 0.1664 \\
\hline LVEDd $(\mathrm{cm}) 6$ months after surgery & $2.04(0.52)$ & $2.1( \pm 0.56)$ & 0.5778 \\
\hline LVESd $(\mathrm{cm}) 6$ months after surgery & $1.38(0.35)$ & $1.43(+0.44)$ & 0.4230 \\
\hline
\end{tabular}


There was a $13 \%$ increase in the odds of having unusual CA pattern for every 0.01 increase in the C-Ratio.

The surgical bypass time was significantly shorter in patients with usual CA pattern compared to those with unusual CA pattern $(P<0.05)$. This reflects the burden of the abnormal CA pattern on the surgical approach. This also affected the medium-term postoperative outcome as patients with minor malalignment had significantly smaller left ventricle end-diastolic dimensions (LVEDd) at 6 months follow-up $(P<0.05)$.

In the literature, there are some studies trying to explain the embryological background behind the rotational abnormalities occurring in patients with TGA. In 1981, De la Cruz and her colleagues $[17,18]$ have suggested that there is no rotation at the infundibular level in both normal and pathological cases. They postulated that TGA is due to a linear rather than a spiral development of the aorto-pulmonary septum, which places the forth aortic arch (the future aorta) in direct connection with the anterior conus situated in the right ventricle. Other studies showed that a clockwise spiraling migration of cells from the right and left secondary heart field is necessary for the proper alignment of the pulmonary outflow in order for it to attain the classical righthanded spiral pattern [19-21]. Bajolle et al. conducted an interesting study and identified the E15.5 transgene which is normally detected at the base of the pulmonary trunk, and lies ventral to the aorta. They proved that in contrast to the normal hearts, TGA hearts had a transgene expression maintained at the base of the pulmonary trunk and positioned dorso-laterally indicating a rotation defect [22]. This confirms the importance of the spiral movement of outflow tract in normal hearts.

Based on the study done by De la Cruz, we contemplate that commissural malalignment could be attributed to the arrest of the spiral rotation of the aorto-pulmonary trunk at varying degrees in patients with TGA. This is supported by the "Infundibular theory" postulated by Goor et al. who suggested that TGA occurs due lack of the normal counter clockwise rotation of the conotruncus. He suggested that TGA occurs due to a defect of the normal infundibular rotation with abnormal resorption or underdevelopment of the subpulmonary conus and abnormal persistence of the subaortic conus [19, 23]. Greater or lesser degree of faulty absorption of the conus leads to the spectrum of TGA [23]. This probably explains why the vast majority $(91 \%)$ of the patients with malalignment in our study had sinus facing the pulmonary valve, and it may also indicate the importance of the spiral movement of the outflow tract, as the rotation arrest of the pulmonary trunk with possible anterior rotation of the aortic truck leads to the sinus facing the pulmonary valve.

The relation between the abnormal CA pattern and the aorto-pulmonary sinus rotation, ie malalignment was investigated in several studies [15, 24-26]. The usual CA pattern is seen in majority of patients with TGA where the RCA is connected to the right posterior facing sinus and the left CA (LCA) is connected to the left facing sinus [15]. However, changes in the position of the aorta may result in abnormal CA pattern [24]. Hutchins et al. found that the normal site of CA connection was determined by the configuration of the root of the great arteries in the embryonic human heart. He indicated that the position of the aorto-pulmonary sinuses affects the CA pattern [25]. Bogers et al. have indicated that the epicardial CA gets connected to the nearest sinus to its origin [26]. This idea of ingrowth of epicardial coronaries towards the neighboring aorta and their subsequent development of a lumen have been discussed in several other studies [15, 24-28]. Embryologic studies showed that the coronary arteries develop after the septation of the aortopulmonary trunk $[24,29]$. Thus, the coronary arteries pierce the nearest site of the aortic sinus after completion of the aorto-pulmonary rotation. Consequently, the CA pattern is dependent on the preceding aorto-pulmonary rotation [24, 29]. Therefore, we believe that the abnormality in the aorto-pulmonary sinus rotation, with an arrest of spirality at some stage, might be the reason behind the commissural malalignment. This arrest of the rotation of the aorto-pulmonary sinus may result also in the unusual pattern of coronary arteries occurring with varying degrees of commissural malalignment.

Similar to other studies, we showed that patients with unusual CA pattern have significantly higher bypass time during the ASO compared to those with usual CA pattern [30]. Nevertheless, there were no serious adverse events postoperatively related to the CA pattern in our patients. Furthermore, there was no difference in the short-term postoperative cardiac function between those with minor or major CM. In a recent study, Altin et al. have suggested that patients with TGA and unusual CA pattern are more prone to ventricular dysfunction, however there was no significant difference in the hospital stay between the patients with usual or unusual CA pattern in the first 6 months post-operatively [30]. They reported that intramural course of coronary arteries and the single RCA were the only unusual CA patterns that have an operative risk factor. In our study we had 5 early post-operative mortalities with no significant relation to the coronary artery pattern ( 3 patients had usual coronary arteries, 1 patient had inverted coronary arteries, and 1 patient had a single left CA). Another patient in our group died pre-operatively who had a usual CA pattern.

\section{Conclusion}

Commissural malalignment in patients with TGA is a good predictor of abnormal CA pattern. The C-Ratio helps to categorize the degree of CM. Patients with C- 
Ratio more than 0.31 are considered as having major $\mathrm{CM}$ and are expected to have unusual CA pattern. CM and unusual CA pattern have negative impact on the outcome of the arterial switch operation.

\section{Acknowledgements}

Not applicable

\begin{abstract}
Authors' contributions
Mohamed Al Nasef, contributed in measuring the D1 and D2 distances and measuring the C-Ratio for all patients, Mohammed H. Alghamdi, Maria L. Bello Valls, Ahmed M. Zahrani, Ali AlAkfash, Obayda M. Diranneya confirmed the anatomy and way of measurment, Husam I. Ardah helped analyze the results statistically Fahad Alhabshan helped supervise the whole project. The authors read and approved the final manuscript.
\end{abstract}

\section{Funding}

Not Applicable.

\section{Availability of data and materials}

The data that support the findings of this study are available from King Abdulaziz Cardiac Centre, but restrictions apply to the availability of these data, which were used under license for the current study, and so are not publicly available. Data are however available from the authors upon reasonable request and with permission of King Abdullah Internal Medical Research Centre.

\section{Ethics approval and consent to participate}

Not Applicable

\section{Consent for publication}

Not Applicable

\section{Competing interests}

Not Applicable.

\section{Author details}

'Cardiac Sciences Department, Ministry of the National Guard - Health Affairs, Riyadh, Saudi Arabia. ${ }^{2}$ Pediatric cardiology department, Salmaniya Medical Complex, Manama, Bahrain. ${ }^{3}$ King Fahad Cardiac Centre, King Saud University, Riyadh, Saudi Arabia. ${ }^{4}$ Department of Pediatric Cardiology, Prince Sultan Cardiac Centre, Riyadh, Saudi Arabia. ${ }^{5}$ Pediatric Cardiology, Prince Sultan Cardiac Center-Qassim MCH, Buraidah, Saudi Arabia. ${ }^{6}$ King Abdullah International Medical Research Center, Riyadh, Saudi Arabia. ${ }^{7}$ Cardiac Sciences, College of Medicine, King Saud bin Abdulaziz University for Health Sciences, Riyadh, Saudi Arabia.

\section{Received: 9 November 2019 Accepted: 28 April 2020}

Published online: 02 October 2020

\section{References}

1. Hoffman Jl, Kaplan S. The incidence of congenital heart disease. J Am Coll Cardiol. 2002;39(12):1890-900.

2. Pasquini $L$, Sanders SP, Parness IA, Colan SD. Diagnosis of coronary artery anatomy by two-dimensional echocardiography in patients with transposition of the great arteries. Circulation. 1987;75(3):557-64.

3. Gremmels DB, Tacy TA, Brook MM, Silverman NH. Accuracy of coronary artery anatomy using two-dimensional echocardiography in d-transposition of great arteries using a two-reviewer method. J Am Soc Echocardiogr. 2004;17(5):454-60.

4. Fundora MP, Aregullin EO, Wernovsky G, et al. Echocardiographic and surgical correlation of coronary artery patterns in transposition of the great arteries. Congenit Heart Dis. 2016;11(6):570-7.

5. Hutter PA, Bennink GB, Ay L, Raes IB, Hitchcock JF, Meijboom EJ. Influence of coronary anatomy and reimplantation on the long-term outcome of the arterial switch. Eur J Cardiothorac Surg. 2000;18(2):207-13.

6. Yacoub MH, Radley-Smith R. Anatomy of the coronary arteries in transposition of the great arteries and methods for their transfer in anatomical correction. Thorax. 1978;33(4):418-24.
7. Day RW, Laks H, Drinkwater DC. The influence of coronary anatomy on the arterial switch operation in neonates. J Thorac Cardiovasc Surg. 1992;104(3):706-12.

8. Wernovsky G, Sanders SP. Coronary artery anatomy and transposition of the great arteries. Coron Artery Dis. 1993:4(2):148-57.

9. Blume ED, Altmann K, Mayer JE, Colan SD, Gauvreau K, Geva T. Evolution of risk factors influencing early mortality of the arterial switch operation. J Am Coll Cardiol. 1999;33(6):1702-9.

10. Pasquali SK, Hasselblad V, Li JS, Kong DF, Sanders SP. Coronary artery pattern and outcome of arterial switch operation for transposition of the great arteries: a meta-analysis. Circulation. 2002;106(20):2575-80.

11. Ismail SR, Kabbani MS, Najm HK, Abusuliman RM, Elbarbary M. Early outcome for the primary arterial switch operation beyond the age of 3 weeks. Pediatr Cardiol. 2010;31(5):663-7.

12. Lacour-Gayet F. Complexity stratification of the arterial switch operation: a second learning curve. Cardiol Young. 2012;22(6):739-44.

13. Kim SJ, Kim WH, Lim C, Oh SS, Kim YM. Commissural malalignment of aortic-pulmonary sinus in complete transposition of great arteries. Ann Thorac Surg. 2003;76(6):1906-10.

14. Lai WW, Geva T, Shirali GS, et al. Guidelines and standards for performance of a pediatric echocardiogram: a report from the task force of the pediatric Council of the American Society of echocardiography. J Am Soc Echocardiogr. 2006:19(12):1413-30.

15. Sim EK, van Son JA, Edwards WD, Julsrud PR, Puga FJ. Coronary artery anatomy in complete transposition of the great arteries. Ann Thorac Surg. 1994;57(4):890-4.

16. Vouhe PR, Haydar A, Ouaknine R, et al. Arterial switch operation: a new technique of coronary transfer. Eur J Cardiothorac Surg. 1994;8(2):74-8.

17. de la Cruz MV, Sanchez Gomez C, Arteaga MM, Arguello C. Experimental study of the development of the truncus and the conus in the chick embryo. J Anat. 1977;123(Pt 3):661-86.

18. de la Cruz MV, Arteaga M, Espino-Vela J, Quero-Jimenez M, Anderson RH, Diaz GF. Complete transposition of the great arteries: types and morphogenesis of ventriculoarterial discordance. Am Heart J. 1981;102(2):271-81.

19. Unolt M, Putotto C, Silvestri LM, et al. Transposition of great arteries: new insights into the pathogenesis. Front Pediatr. 2013;1:11.

20. Takahashi M, Terasako Y, Yanagawa N, Kai M, Yamagishi T, Nakajima Y. Myocardial progenitors in the pharyngeal regions migrate to distinct conotruncal regions. Dev Dyn. 2012;241(2):284-93.

21. Ward C, Stadt H, Hutson M, Kirby ML. Ablation of the secondary heart field leads to tetralogy of Fallot and pulmonary atresia. Dev Biol. 2005;284(1):72-83.

22. Bajolle F, Zaffran S, Kelly RG, et al. Rotation of the myocardial wall of the outflow tract is implicated in the normal positioning of the great arteries. Circ Res. 2006;98(3):421-8.

23. Goor DA, Edwards JE. The spectrum of transposition of the great arteries: with specific reference to developmental anatomy of the conus. Circulation. 1973:48(2):406-15.

24. Chiu IS, Chu SH, Wang JK, et al. Evolution of coronary artery pattern according to short-axis aortopulmonary rotation: a new categorization for complete transposition of the great arteries. J Am Coll Cardiol. 1995;26(1):250-8.

25. Hutchins GM, Kessler-Hanna A, Moore GW. Development of the coronary arteries in the embryonic human heart. Circulation. 1988;77(6):1250-7.

26. Bogers AJ, Gittenberger-de Groot AC, Poelmann RE, Peault BM, Huysmans HA. Development of the origin of the coronary arteries, a matter of ingrowth or outgrowth? Anat Embryol. 1989;180(5):437-41.

27. Poelmann RE, Gittenberger-de Groot AC, Mentink MM, Bokenkamp R, Hogers B. Development of the cardiac coronary vascular endothelium, studied with antiendothelial antibodies, in chicken-quail chimeras. Circ Res. 1993;73(3):559-68.

28. Vrancken Peeters MP, Gittenberger-de Groot AC, Mentink MM, Hungerford JE, Little CD, Poelmann RE. The development of the coronary vessels and their differentiation into arteries and veins in the embryonic quail heart. Dev Dyn. 1997;208(3):338-48.

29. Angelini P. Normal and anomalous coronary arteries: definitions and classification. Am Heart J. 1989:117(2):418-34.

30. Altin FH, Sengul FS, Yildiz $\mathrm{O}$, et al. Impact of coronary artery anatomy in arterial switch procedure on early mortality and morbidity. Congenit Heart Dis. 2016;11(2):115-21

\section{Publisher's Note}

Springer Nature remains neutral with regard to jurisdictional claims in published maps and institutional affiliations. 